\title{
REPORT OF THE TREASURER FOR THE PERIOD DECEMBER 1, 1935 TO NOVEMBER 30, 1936
}

\author{
BALANCE SHEET
}

November 30, 1936

ASSETS

\section{CASH IN BANKS:}

Corn Exchange Bank Trust Company ... .\$ 8,932.83

Bank for Savings ................ 2,673.50

Providence Institution for Savings ..... 5, 158.88

Union Dime Savings Bank ......... 5,752.38

INVESTMENTS (Value based on market quotation as of November $30,1936, \$ 92,866.95$.)

RESERVES AND SURPLUS

Reserves :

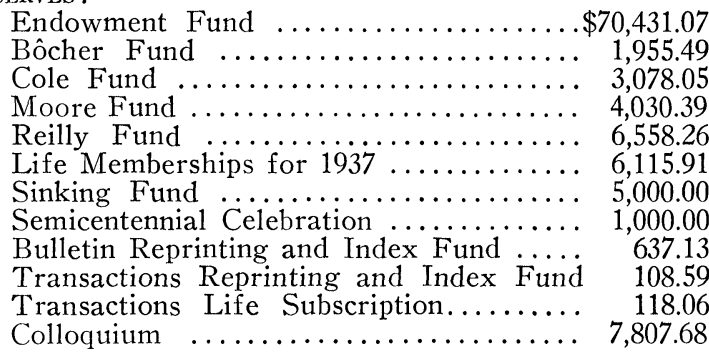

Surplus at November 30, 1936

Total Reserves and Surplus

STATEMENT OF GENERAL RECEIPTS AND GENERAL DISBURSEMENTS

GenerAl ReCEIPTS :

Dues from Ordinary Memberships $\$ 12,188.99$

Dues from Contributing Memberships ................ 1,206.00

Dues from Institutional Memberships ............. 6,180.00

Initiation Fees ........................... ${ }_{769} .37$

Interest Income from Endowment Fund and General

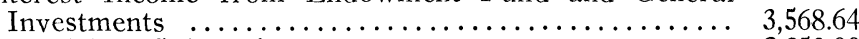

Rockefeller Subvention $\ldots \ldots \ldots \ldots \ldots \ldots \ldots \ldots \ldots \ldots, 2,250.00$

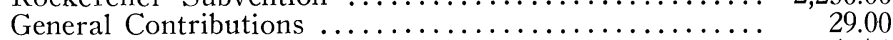

Miscellaneous ............................... 653.72

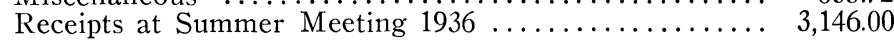

Total General Receipts

General Disbursements :

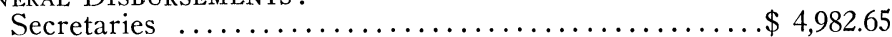

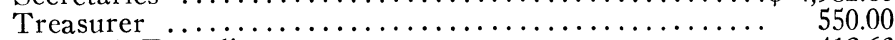

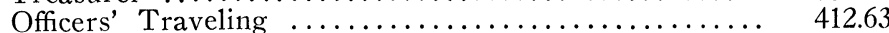

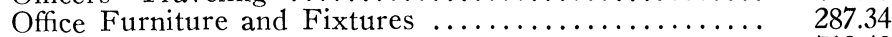

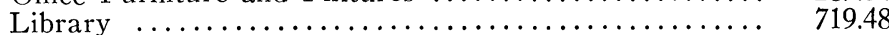

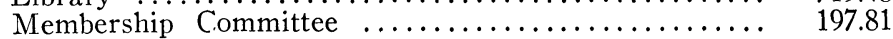


Semicentennial Committee $\ldots \ldots \ldots \ldots \ldots \ldots \ldots \ldots \ldots . \ldots \ldots$

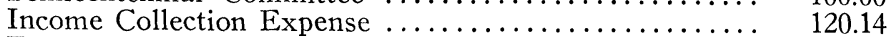

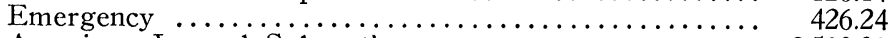

American Journal Subvention $\ldots \ldots \ldots \ldots \ldots \ldots \ldots \ldots \ldots, 2,500.00$

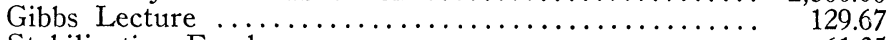

Stabilization Fund ....................................... ${ }^{6} 61.35$

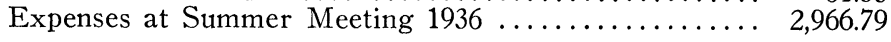

Total General Disbursements .............. 13,454.10

Excess of General Receipts over General Disbursements $\$ 16,537.62$

Surplus at December 3, $1935 \ldots \ldots \ldots \ldots \ldots \ldots \ldots \ldots \ldots \ldots \ldots \ldots$

Additions :

Balances at December 3, 1935 Transferred to Surplus . .\$4,910.26

Adjustment of Life Membership Reserve ........... 338.70

Adjustment of Transactions Life Subscription Reserve 2.63

Excess of General Receipts over General Disbursements

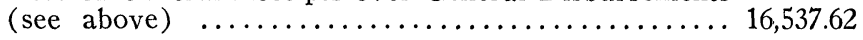

Total

Deductions :

Appropriation to Bulletin Account .............. \$ 8,097.16

Appropriation to List of Members Account .......... 400.00

Appropriation to Transactions Account ........... 2,280.70

Appropriation to Colloquium Account ................ 1,250.00

Appropriation to Semicentennial Fund ............. 1,000.00

Appropriation to Sinking Fund $\ldots \ldots \ldots \ldots \ldots \ldots \ldots \ldots \ldots, 3,500.00$

Accrued Interest Purchased $\ldots \ldots \ldots \ldots \ldots \ldots \ldots \ldots . \quad 136.38$

Surplus at November $30,1936 \ldots \ldots \ldots \ldots \ldots \ldots \ldots \ldots . \overline{\$ 14,962.11}$

BULLETIN

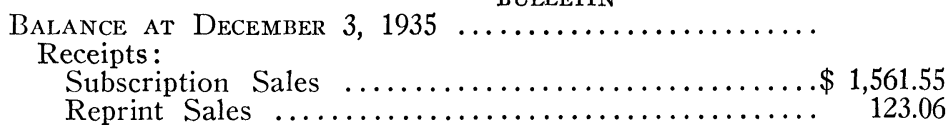

Appropriation from General Receipts .............. 8,097.16

Total .............................. $\overline{\$ 11,701.69}$

Disbursements:
Printing (2 numbers for 1935,10 for 1936$) \ldots \ldots \ldots . \$ 6,575.72$

Salaries, Annuity and Pension ................. 2,252.26

Miscellaneous-Office Furniture, Supplies, etc. ....... 839.95

Editors' Traveling ........................... $\quad 80.00$

Circulars and Advertising . ......................... 33.84

Lapse of Unexpended Balance at December 3, 1935 .. $1,919.92$

$11,701.69$

\section{BULLETIN-LIST OF MEMBERS}

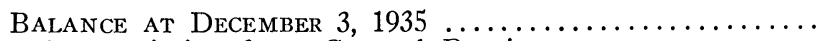

Appropriation from General Receipts $\ldots \ldots \ldots \ldots \ldots \ldots \ldots$.

$\$ \quad 400.00$

400.00

Total

$\$ 800.00$

Disbursements :

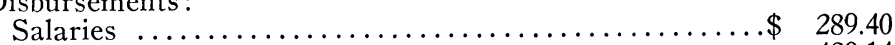

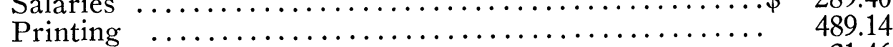

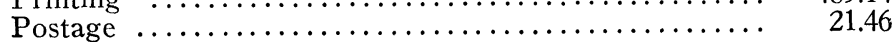

800.00 
BULLETIN REPRINTING AND INDEX

Balance at December 3, $1935 \ldots \ldots \ldots \ldots \ldots \ldots \ldots \ldots$

Receipts :

Back Volume Sales

$\$ 1,312.48$

Total

520.86

$\$ 1,833.34$

Disbursements :

Reprinting

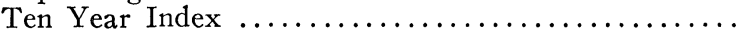

420.93

Clerical, etc.

Balance at November 30, 1936

$\begin{array}{r}1,196.21 \\ \$ \quad 637.13 \\ \hline\end{array}$

TRANSACTIONS

Balance at December $3,1935 \ldots \ldots \ldots \ldots \ldots \ldots \ldots \ldots \ldots$

Receipts :

Subscription Sales

$\$ 2,679.31$

Back Volume Sale

$\$ 4,299.47$

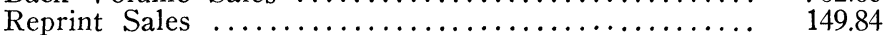

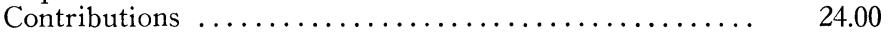

Appropriation from General Receipts ............. 2,280.70

Total ........................... $\overline{\$ 10,195.97}$

Disbursements :

Printing (5 numbers for 1936) $\ldots \ldots \ldots \ldots \ldots \ldots \ldots \ldots 5,485.05$

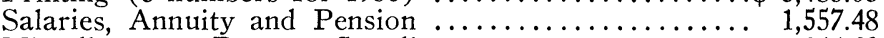

Miscellaneous-Postage, Supplies, etc. ............ 364.29

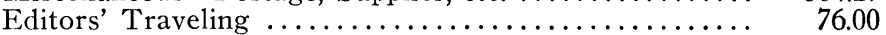

Circulars and Advertising .................. 33.84

Lapse of Unexpended Balance at December $3,1935 \ldots \ldots 2,679.31$

TRANSACTIONS REPRINTING AND INDEX

Balance at December 3, 1935

$\$ \quad 459.14$

Disbursements :

Reprinting

Balance at November 30, 1936

$\$ 108.59$

COLLOQUIUM

Balance at December 3, 1935

$\$ 6,785.11$

Receipts :

Sales of Colloquium Volumes

Sales of Colloquium Volume 16 (Bliss) for National

Research Council ........................... 186.37

Colloquium Lecture Fees ..................... 440.00

Appropriation from General Receipts by Order of Trustee

$1,250.00$

Total

Disbursements :

Printing (Volume 20) ...................... 2,734.35

Salaries $(. \ldots \ldots \ldots \ldots \ldots \ldots \ldots \ldots \ldots \ldots \ldots \ldots \ldots, 725.00$

Miscellaneous-Shipping, Supplies, etc. (including cost of shipping Volume 16 for National Research Council)

Circulars and Advertising.

Customs and Duty 
Payment to National Research Council for Volume 16

(Bliss) .................................. 156.37

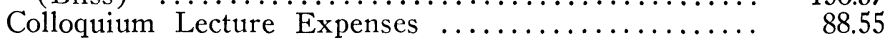

Balance at November $30,1936 \ldots \ldots \ldots \ldots \ldots \ldots \ldots \ldots . \overline{\$ 7,807.68}$

\begin{tabular}{|c|c|c|c|}
\hline ENDOWMENT FUND & Total & Investments & Cash \\
\hline $\begin{array}{l}\text { Balance at December 3, } 1935 \text {. } \\
\text { Additions. }\end{array}$ & $. \$ 73,183.87$ & $\$ 72,568.26$ & $\$ 615.61$ \\
\hline $\begin{array}{l}\text { Additions: } \\
\text { Income and Gifts } \ldots \ldots .\end{array}$ & $2,915.60$ & & $2,915.60$ \\
\hline$\underset{\text { Total }}{\text {. . }}$ & $\$ 76,099.47$ & $\$ 72,568.26$ & $\$ 3,531.21$ \\
\hline $\begin{array}{l}\text { Transferred to General Receipts } \ldots \ldots \\
\text { Accrued Interest Purchased } \ldots . . . \ldots\end{array}$ & $\begin{array}{l}2,890.60 \\
2,777.80\end{array}$ & $2,777.80$ & $2,890.60$ \\
\hline Balance at November 30, 1936 . & $. \$ 70,431.07$ & $\$ 69,790.46$ & $\$ 640.61$ \\
\hline $\begin{array}{l}\text { BôCHER Fund } \\
\text { Balance at December 3, } 1935\end{array}$ & . $\$ 1,892.40$ & $\$ 1,663.60$ & $\$ 228.80$ \\
\hline $\begin{array}{l}\text { Additions : } \\
\text { Income } \ldots \ldots \ldots \ldots . . . . . . \\
\text { Redemption of } \text { Bond } \ldots\end{array}$ & $\begin{array}{r}71.94 \\
169.76\end{array}$ & & $\begin{array}{r}71.94 \\
169.76\end{array}$ \\
\hline $\begin{array}{r}\text { Total } \\
\text { Dedurtion }\end{array}$ & $. \$ 2,134.10$ & $\$ 1,663.60$ & $\$ 470.50$ \\
\hline $\begin{array}{l}\text { Deductions: } \\
\text { Accrued Interest Purchased ... } \\
\text { Redemption of Bond ........ }\end{array}$ & $\begin{array}{r}8.85 \\
169.76\end{array}$ & $\begin{array}{r}8.85 \\
169.76\end{array}$ & \\
\hline Balance at November 30, 1936 & $. \$ 1,955.49$ & $\$ 1,484.99$ & $\$ 470.50$ \\
\hline
\end{tabular}

(Principal \$1,188.00; Income \$767.49.)

Cole Fund

Balance at December 3, 1935

Additions :

Income ............................... 113.94

Redemption of Bond .................... $169.76 \quad 169.76$

Total $\ldots \ldots \ldots \ldots \ldots \ldots \ldots \ldots \ldots \ldots . \overline{\$ 3,255.33} \overline{\$ 2,523.02} \overline{\$ 732.31}$

Deductions :

Accrued Interest Purchased $\ldots . \ldots \ldots \ldots . \quad 7.52 \quad 7.52$

Redemption of Bond .................. $169.76 \quad 169.76$

Balance at November 30,1936 ........... $\overline{\$ 3,078.05} \overline{\$ 2,345.74} \overline{\$ 732.31}$

(Principal \$2,093.13; Income \$984.92.)

MoORE Fund

Balance at December 3, $1935 \ldots \ldots \ldots \ldots \ldots \ldots$. $3,918.83$ \$2,973.81 \$ 945.02

Addition:

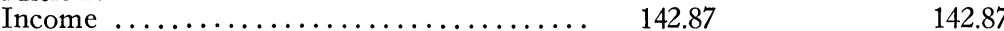

Total $\ldots \ldots \ldots \ldots \ldots \ldots \ldots \ldots \ldots \ldots . \overline{\$ 4,061.70} \overline{\$ 2,973.81} \overline{\$ 1,087.89}$ 


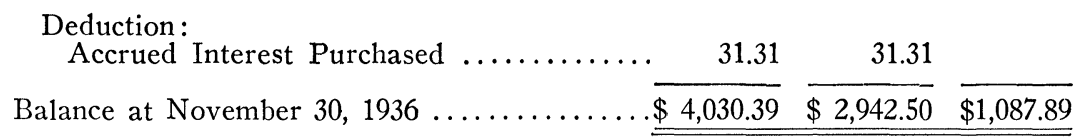

(Principal \$2,100.62; Income $\$ 1,929.77$.)

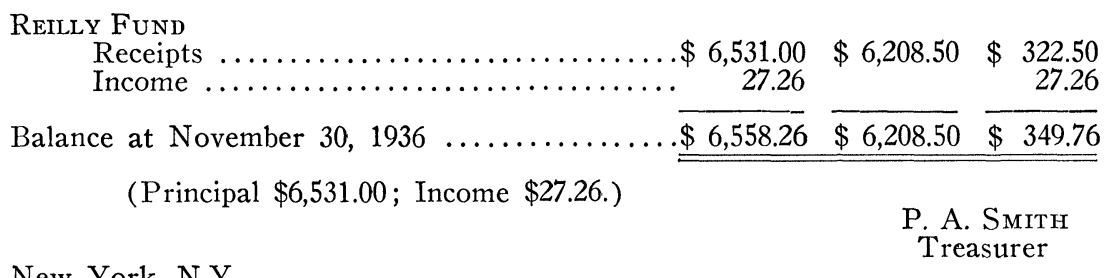

November 30,1936

\section{REPORT OF THE AUDITING COMMITTEE}

\section{6}

We, the undersigned Auditing Committee, appointed by the American Mathematical Society, have this day audited the accounts of the Treasurer and in our opinion the following statement is correct:

Cash Balance at December 3, 1935 :

Corn Exchange Bank Trust Company

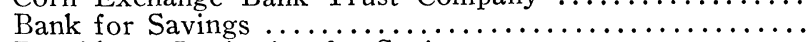

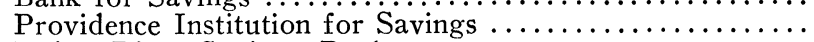

Union Dime Savings Bank

General ..................................... \$3,400.72

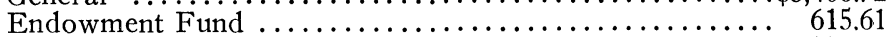

Bôcher Fund .................................. 228.80

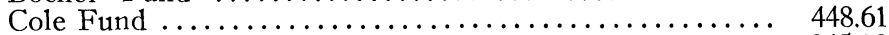

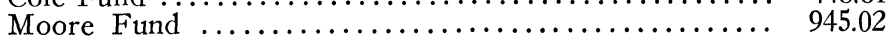

Cash Receipts-December 3, 1935 to November 30, 1936 ... $\quad 39,892.66$

Interest on Savings Accounts ...................... 293.00

Total .............................. $\overline{\$ 60,201.57}$

Cash Disbursements-December 3, 1935 to November 30, $1936 \quad 37,683.98$

Cash Balance at November 30, 1936 :

Corn Exchange Bank Trust Company .............\$8,932.83

Bank for Savings ................................ 2,673.50

Providence Institution for Savings $\ldots \ldots \ldots \ldots \ldots \ldots \ldots \ldots \ldots \ldots \ldots \ldots \ldots \ldots \ldots \ldots, 158.88$

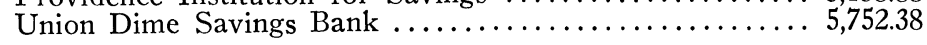

S. A. JofFe

New York, N.Y.

A, E. Meder, JR.

December 11, 1936 